\title{
Association of gene polymorphisms of KLK3 and prostate cancer: A meta-analysis
}

\author{
${ }^{*}$ Huifeng $\mathrm{Li}^{1, \mathrm{C}}$, ${ }^{*}$ Xiawei Fei ${ }^{1, \mathrm{~A}, \mathrm{C}}$, Yanting Shen ${ }^{2, \mathrm{~A}}$, Zhenqi Wu${ }^{1, \mathrm{~F}}$ \\ ${ }^{1}$ Department of Urology, Qingpu Branch of Zhongshan Hospital affiliated to Fudan University, Shanghai, China \\ ${ }^{2}$ School of Biological Science and Medical Engineering, Southeast University, Nanjing, China \\ A - research concept and design; $\mathrm{B}$ - collection and/or assembly of data; $\mathrm{C}$ - data analysis and interpretation; \\ $D$ - writing the article; $E$ - critical revision of the article; $F$ - final approval of the article
}

\section{Address for correspondence \\ Zhenqi Wu}

E-mail:zhenqi_wu1976@sina.com

\section{Funding sources}

The meta-analysis was supported by the Science and Technology Development Foundation of Qingpu (project No. QKY2017-12) and by the Qingpu Branch of Zhongshan Hospital affiliated to Fudan University Research Project (project No. QY2017-05).

Conflict of interest

None declared

*Huifeng Li and Xiawei Fei contributed equally to this work.

Received on September 11, 2018

Reviewed on 0ctober 14, 2018

Accepted on April 30, 2020

Published online on September 1, 2020

\begin{abstract}
Previous studies have suggested that prostate-specific antigen (PSA) plays a role in the etiology of prostate cancer (PCa), and that polymorphisms of KLK3 may be associated with PCa. However, these results were conflicting. Therefore, we performed a meta-analysis to illuminate this problem. We searched the PubMed and Web of Science databases. Ten single nucleotide polymorphisms (SNPS) were involved in this meta-analysis. The pooled results showed that the minor alleles of rs1058205, rs2735839, rs174776, rs17632542, rs266849, is266878, and rs2569735 were significantly associated with PCa. Compared to genotypes of the common homozygotes, the heterozygous genotypes of rs1058205, rs2735839, rs174776, rs 17632542, rs266849, and rs266878 were significantly associated with PCa, as well as the homozygous genotypes of rs1058205, rs2735839, rs 17632542, rs266878, rs266876, and rs2569735. Only rs2735839 was involved in the Gleason score (GS). The pooled results showed that when compared with GS $\geq 8$ PCa, the A-allele was the protective factor for $G S<7 P$ Ca. It was also a protective factor for $G S \geq 4+3$ when compared to $G S \leq 3+4 P$ Ca. A strong association was observed between PCa and rs1058205, rs2735839, rs266882, rs 174776, rs 17632542, rs266849, rs266878, rs266876, rs 1058274, and rs2569735. The G-allele of rs2735839 was a risk factor for GS $<7 \mathrm{PCa}$ when compared with the $G S \geq 8 P C$ a, as well as for the $G S \geq 4+3$ when compared to the $G S \leq 3+4 P C$. Therefore, these SNPs may be valuable as biomarkers for PCa in the future.
\end{abstract}

Key words: KLK3, prostate-specific antigen, polymorphisms, prostate cancer, meta-analysis

Cite as

Li H, Fei X, Shen Y, Wu Z. Association of gene polymorphisms of KLK3 and prostate cancer: A meta-analysis. Adv Clin Exp Med. 2020;29(8):1001-1009. doi:10.17219/acem/121521

DOI

10.17219/acem/121521

\section{Copyright}

OCopyright by Author(s)

This is an article distributed under the terms of the

Creative Commons Attribution 3.0 Unported (CC BY 3.0)

(https://creativecommons.org/licenses/by/3.0/) 
Prostate cancer $(\mathrm{PCa})$ is the $2^{\text {nd }}$ most frequently diagnosed cancer in men around the world, and one of the leading causes of cancer death among men of all races. ${ }^{1}$ With the aging of the population and the improvement of living conditions in recent years, the incidence of PCa has been increasing every year. ${ }^{2}$ Serum levels of prostate-specific antigen (PSA) are widely used for screening for PCa. The PSA levels are known to be influenced by genetic components: Around $40-45 \%$ of the variance in PSA is thought to be explained by genetic components. ${ }^{3,4}$ Previous studies have revealed that kallikrein $3(K L K 3)$ is the strongest genetic factor to influence levels of PSA, and its single nucleotide polymorphism (SNP) loci have been shown to be associated with $\mathrm{PCa}{ }^{5-7}$

The KLK3 is located on chromosome 19q13.33, which encodes PSA and is a member of the serine protease kallikrein family. We searched the PubMed and Web of Science databases without language restrictions up to January 8, 2018, for relevant studies about the association of the SNPs of $K L K 3$ and PCa. We found that nearly 59 SNP loci were mentioned in studies in these databases, and among them, 21 SNP loci were involved in more than 2 studies (Fig. 1). However, these results were conflicting and there was still a lack of any relevant comprehensive analysis to clarify the confusion.

Therefore, in this study, we performed a literature review and a meta-analysis to explore the association between the risk of PCa and the 21 SNP loci of $K L K 3$ that were mentioned in more than 2 studies.

\section{Material and methods}

\section{Search strategy}

We searched the PubMed and Web of Science databases through September, 2018, without language restrictions, for relevant studies about associations of the SNPs of $K L K 3$ and PCa. The search term was ((KLK3) AND ((single nucleotide polymorphism) OR SNP))) AND ((prostate cancer) OR PSA).

\section{Inclusion/exclusion criteria}

The title, abstract and full text of the candidate studies were independently screened by 2 reviewers. A study was included when all of the following criteria were met: 1) Nonfamilial studies that examined the association between SNPs of $K L K 3$ and PCa were included; 2) studies that had complete data or data that could be used to calculate an odds ratio (OR) and a 95\% confidence interval (95\% CI) were included; 3) studies that had incomplete data were excluded.

\section{Data extraction}

Information was carefully extracted from all the eligible publications by 2 independent reviewers ( $\mathrm{Li}$ and Fei), based on the aforementioned inclusion criteria. Any disagreements were arbitrated by discussion with a $3^{\text {rd }}$ reviewer (Shen). The following data were collected from each study: the $1^{\text {st }}$ author's surname, the year of publication, the country, the laboratory methods used to detect $K L K 3$ polymorphisms, and the number of cases and controls.

\section{Quality assessment}

We used the Newcastle-Ottawa scale (NOS) to assess the quality of each eligible study. The NOS contains 8 items: 1) The cases were independently validated;2) Cases were representative of a population; 3) There were community controls; 4) The controls had no history of PCa; 5A) The study was controlled for age; 5B) The study was controlled for additional factors; 6) Exposure was ascertained by blinded interview or record; 7) The same method of ascertainment was used for both the cases and the controls; 8) The nonresponse rate was the same for the cases and the controls. When a study fulfilled 1 criterion, it got 1 score. The NOS is arranged from 0 up to 9 scores, and a study is considered high quality if it gets more than 4 scores.

\section{Statistical analysis}

The strength of the association between $K L K 3$ polymorphism and the risk of PCa was shown using an OR with a 95\% CI. If a study just provided the frequency (assumption: the frequency of allele 1 or genotype 1 in the case group was A; the frequency of allele 2 or genotype 2 in the case group was $\mathrm{B}$; the frequency of allele 1 or genotype 1 in the control group was $C$; the frequency of allele 2 or genotype 2 in the control group was D), we used the formulas "OR $=(\mathrm{A} / \mathrm{B}) /(\mathrm{C} / \mathrm{D})$ " and "95\% CI of In OR $=$ In $(\mathrm{OR}) \pm 1.96(1 /$ $\mathrm{A}+1 / \mathrm{B}+1 / \mathrm{C}+1 / \mathrm{D})^{0.5}$ " to calculate the OR and its $95 \% \mathrm{CI}$.

The statistical significance of the pooled OR was assessed with a Z-test, and a p-value of 0.05 was considered significant. A $X^{2}$-based Q-test was conducted to measure the heterogeneity of the eligible studies, and the heterogeneity was considered significant if the p-value for the heterogeneity test was 0.05 . A sensitivity analysis in which 1 study was excluded at a time was conducted to evaluate the influence of an individual study on the results. Begg's funnel plot and Egger's regression test were used to evaluate the publication bias (no publication bias was indicated by a two-sided $\mathrm{p}$-value $\geq 0.05$ ). All the analyses were conducted using Stata v. 11.0 software (StataCorp LLC, College Station, USA), and a two-sided $\mathrm{p}$-value $\geq 0.05$ indicated no significance.

\section{Results}

\section{Literature search}

The study selection process is shown in Fig. 2. The primary literature search identified 45 studies. After the titles and abstracts were screened, 13 studies were excluded: 


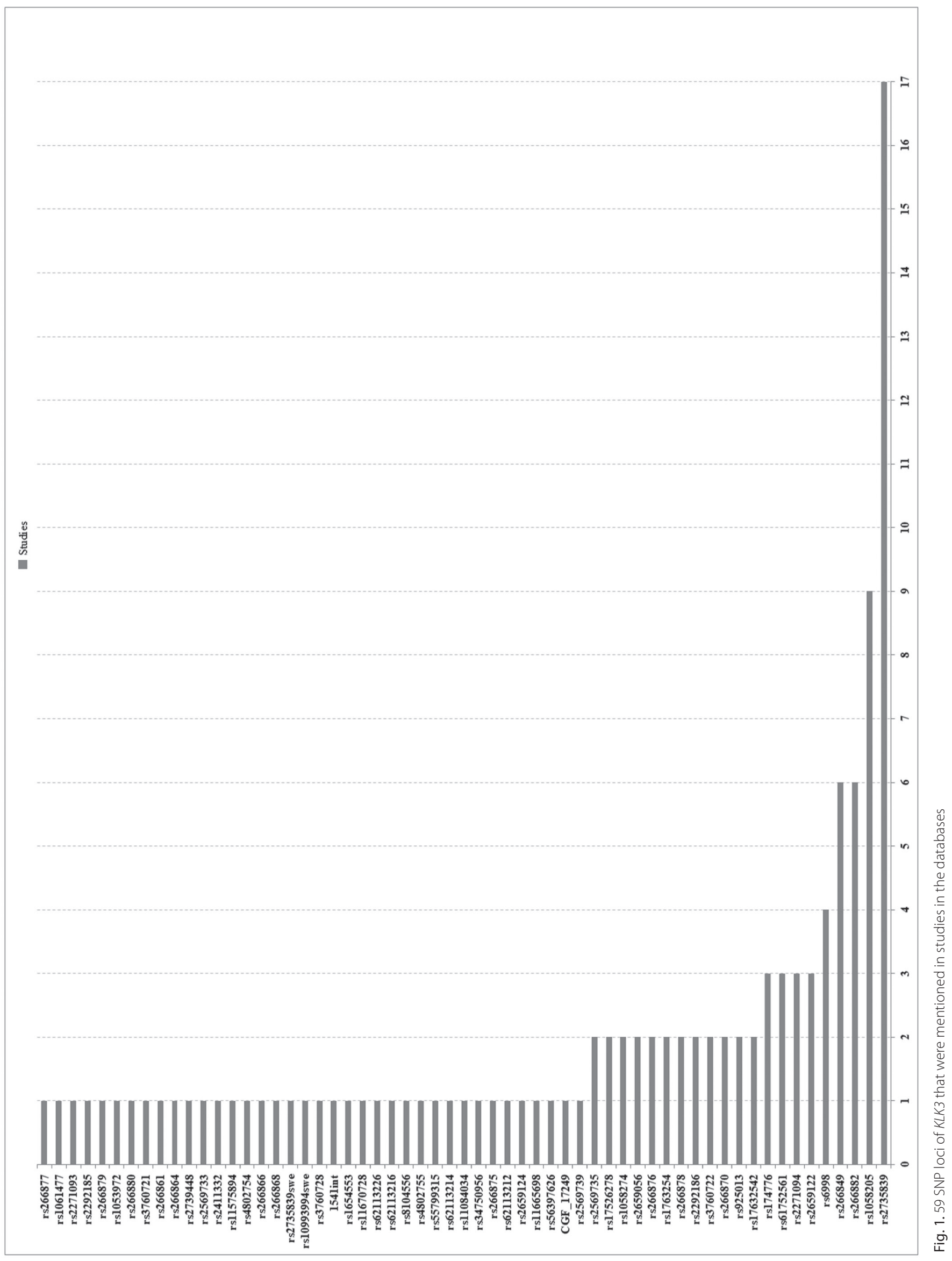




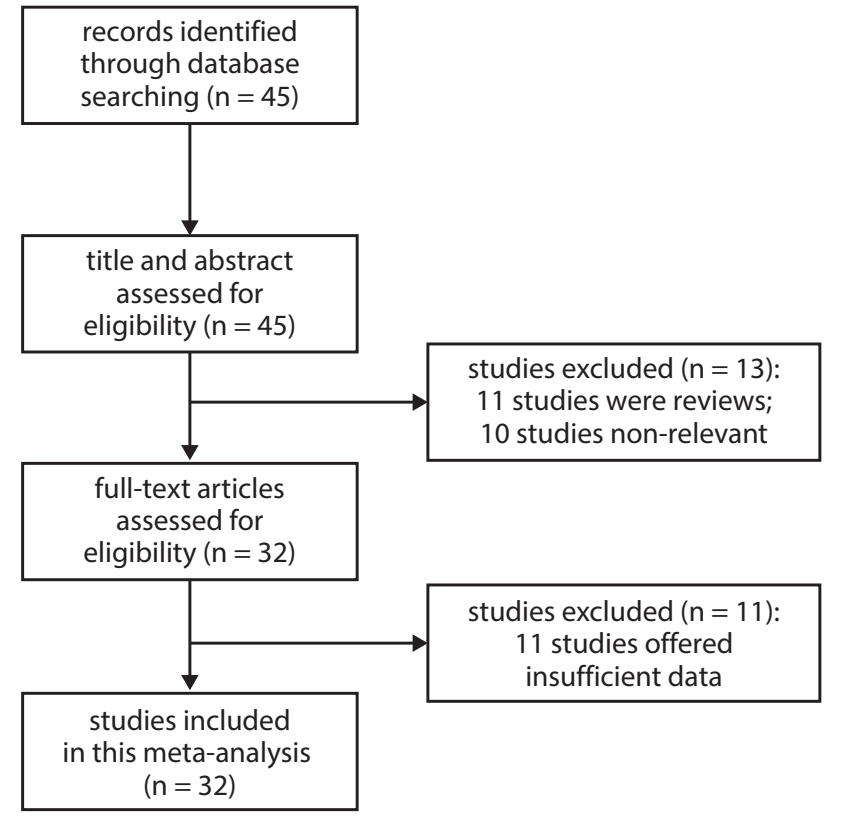

Fig. 2. The study selection process
3 were reviews and 10 were irrelevant studies. The full texts of the remaining 32 studies were then evaluated. As a result, 12 studies were excluded because of useless data and 21 studies were included in the meta-analysis. ${ }^{8-28}$ The 21 eligible studies were assessed with the NOS (Table 1). Each had a score more than 4 , which means that all the studies were of high quality.

\section{Meta-analysis of associations between SNPs and PCa risk}

We found that 10 SNP loci were available to perform a meta-analysis to illuminate associations between the SNPs of KLK3 and PCa risk. They were rs1058205, rs2735839, rs266882, rs174776, rs17632542, rs266849, rs266878, rs266876 rs1058274, and rs2569735..$^{8-10,12,14,16-}$ 22,25-28 Their genetic information is presented in Table 2. The pooled results are shown in Table 3.

For the alleles, we found that except rs266882, rs266876 and rs1058274, the remaining 7 SNP loci were significantly

Table 1. Characteristics and quality assessment of eligible studies in the meta-analysis

\begin{tabular}{|c|c|c|c|c|c|c|c|c|c|c|c|c|c|}
\hline \multirow{2}{*}{ Fist author } & \multirow{2}{*}{ Patients } & \multirow{2}{*}{ Detection method } & \multirow{2}{*}{ Year } & \multicolumn{9}{|c|}{ Quality indicators from NOS } & \multirow{2}{*}{ Score } \\
\hline & & & & 1 & 2 & 3 & 4 & $5 A$ & $5 \mathrm{~B}$ & 6 & 7 & 8 & \\
\hline Choe $\mathrm{EK}^{8}$ & Korean & genotyping arrays & 2017 & yes & yes & no & yes & no & no & yes & yes & yes & 6 \\
\hline Chen $\mathrm{C}^{9}$ & Chinese & PCR-HRM & 2017 & yes & yes & no & yes & yes & yes & yes & yes & yes & 8 \\
\hline Stegeman $\mathrm{S}^{10}$ & European & $\begin{array}{l}\text { Illumina Infinium } \\
\text { Array }\end{array}$ & 2015 & yes & yes & no & yes & no & no & yes & yes & yes & 6 \\
\hline $\mathrm{Hu} \mathrm{J} \mathrm{J}^{12}$ & Chinese & TaqMan/MGB Assay & 2014 & yes & yes & yes & yes & no & no & yes & yes & yes & 7 \\
\hline Shui IM ${ }^{13}$ & European & TaqMan Assay & 2014 & yes & yes & no & yes & no & no & yes & yes & yes & 6 \\
\hline Wang $N^{14}$ & Chinese & PCR-HRM & 2013 & yes & yes & no & yes & no & no & yes & yes & yes & 6 \\
\hline Soni $A^{15}$ & India & PCR-RFLP & 2012 & yes & yes & no & yes & no & no & yes & yes & yes & 6 \\
\hline Kwon EM ${ }^{16}$ & $\begin{array}{c}\text { Caucasian and African } \\
\text { American men }\end{array}$ & genotyping arrays & 2012 & yes & yes & yes & yes & yes & no & yes & yes & yes & 8 \\
\hline Kote-Jarai $Z^{17}$ & UK/Australian & genotyping arrays & 2011 & yes & yes & no & yes & no & no & yes & yes & yes & 6 \\
\hline Penney $\mathrm{KL}^{18}$ & American & $\begin{array}{l}\text { Sequenom } \\
\text { technology }\end{array}$ & 2011 & yes & yes & no & yes & no & no & yes & yes & yes & 6 \\
\hline Lindstrom $\mathrm{S}^{19}$ & European & TaqMan Assay & 2011 & yes & yes & no & yes & no & no & yes & yes & yes & 6 \\
\hline Ciampa J20 & European & Illumina Chips & 2011 & yes & yes & no & yes & no & no & yes & yes & yes & 6 \\
\hline Parikh $\mathrm{H}^{21}$ & European & TaqMan Assays & 2011 & yes & yes & no & yes & no & no & yes & yes & yes & 6 \\
\hline Gudmundsson $J^{22}$ & Icelandic & Illumina Chips & 2010 & yes & yes & no & yes & yes & no & yes & yes & yes & 7 \\
\hline Gallagher $D J^{23}$ & $\begin{array}{c}\text { Ashkenazi Jewish } \\
\text { ancestry }\end{array}$ & $\begin{array}{l}\text { Mass ARRAY QGE } \\
\text { iPLEX System }\end{array}$ & 2010 & yes & yes & no & yes & no & no & yes & yes & yes & 6 \\
\hline Kader $\mathrm{AK}^{24}$ & European & $\begin{array}{l}\text { Mass ARRAY QGE } \\
\text { iPLEX System }\end{array}$ & 2009 & yes & yes & no & yes & no & no & yes & yes & yes & 6 \\
\hline$X u J^{25}$ & European & $\begin{array}{l}\text { Mass ARRAY QGE } \\
\text { iPLEX System }\end{array}$ & 2008 & yes & yes & no & yes & no & no & yes & yes & yes & 6 \\
\hline Eeles RA ${ }^{26}$ & UK and Australia & sequencing & 2008 & yes & yes & no & yes & no & no & yes & yes & yes & 6 \\
\hline Lai J27 & Caucasian men & PCR-RFLP & 2007 & yes & yes & no & yes & no & no & yes & yes & yes & 6 \\
\hline Cicek MS ${ }^{28}$ & American & PCR-RFLP & 2005 & yes & yes & no & yes & no & no & yes & yes & yes & 6 \\
\hline
\end{tabular}

PCR-HRM - high-resolution melting curve polymerase chain reaction method; PCR-RFLP - PCR-restriction fragment length polymorphism; NOS - Newcastle-Ottawa scale. 
Table 2. Genetic information for 10 SNPs of KLK3

\begin{tabular}{|c|c|c|c|c|c|c|}
\hline \multirow{2}{*}{ SNP } & \multirow{2}{*}{ Chromosome $^{\mathrm{a}}$} & \multirow{2}{*}{ Functional consequence $^{a}$} & \multicolumn{2}{|c|}{ Position (bp) $)^{a}$} & \multirow{2}{*}{ Minor allele } & \multirow{2}{*}{ Major allele } \\
\hline & & & GRCh38.p7 & GRCh37.p13 & & \\
\hline rs1058205 & 19:50860142 & URT variant 3 prime & 50860142 & 51363398 & Callele & Tallele \\
\hline rs2735839 & 19:50861367 & downstream & 50861367 & 51364623 & A allele & Gallele \\
\hline rs266882 & $19: 50854757$ & upstream variant $2 \mathrm{~KB}$ & 50854757 & 51358013 & A allele & G allele \\
\hline rs174776 & 19:50856596 & intron variant & 50856596 & 51359852 & Tallele & Callele \\
\hline rs17632542 & 19:50858501 & missense & 50858501 & 51361757 & Tallele & Callele \\
\hline rs266849 & $19: 50845834$ & intron variant & 50845834 & 51349090 & G allele & A allele \\
\hline rs266878 & 19:50855858 & intron variant & 50855858 & 51359114 & G allele & Callele \\
\hline rs266876 & 19:50857562 & intron variant & 50857562 & 51360818 & Callele & Tallele \\
\hline rs1058274 & 19:50860192 & URT variant 3 prime & 50860192 & 51363448 & G allele & A allele \\
\hline rs2569735 & $19: 50861013$ & downstream variant 500B & 50861013 & 51364269 & A allele & Gallele \\
\hline
\end{tabular}

a The information was provided by the dbSNP database (http://www.ncbi.nIm.nih.gov/SNP/); URT - untranslated regions; SNP - single nucleotide polymorphism; KLK3 - kallikrein 3.

Table 3. Meta-analysis of associations between SNPs and PCa risk

\begin{tabular}{|c|c|c|c|c|c|c|c|c|}
\hline \multirow{2}{*}{ SNP } & \multirow{2}{*}{ Number of studies } & \multicolumn{3}{|c|}{ Test for overall effect } & \multicolumn{2}{|c|}{ Test for heterogeneity } & \multicolumn{2}{|c|}{ Test for publish bias } \\
\hline & & OR $(95 \% \mathrm{Cl})$ & Z-score & p-value & $1^{2}$ & $\mathrm{p}$-value & $P_{\text {egger's }}$ & $P_{\text {begg's }}$ \\
\hline \multicolumn{9}{|c|}{ rs1058205 } \\
\hline Callele vs T allele & $\begin{array}{c}8[9,10,16-18,21] \\
\text { a7 }[9,10,16-18,21]\end{array}$ & $\begin{array}{l}0.79(0.73 \sim 0.87) \\
0.85(0.82 \sim 0.88)\end{array}$ & $\begin{array}{l}5.09 \\
8.81\end{array}$ & $\begin{array}{l}<0.001 \\
<0.001\end{array}$ & $\begin{array}{l}83.2 \% \\
10.8 \%\end{array}$ & $\begin{array}{c}<0.001 \\
0.347\end{array}$ & $\begin{array}{c}- \\
0.085\end{array}$ & $\begin{array}{c}- \\
0.133\end{array}$ \\
\hline TC vs TT & $\begin{array}{c}7[9,10,17,18,21] \\
\text { a6 }[9,10,17,18,21]\end{array}$ & $\begin{array}{l}0.79(0.72 \sim 0.86) \\
0.84(0.80 \sim 0.88)\end{array}$ & $\begin{array}{l}5.08 \\
7.87\end{array}$ & $\begin{array}{l}<0.001 \\
<0.001\end{array}$ & $\begin{array}{l}77.6 \% \\
15.8 \%\end{array}$ & $\begin{array}{c}<0.001 \\
0.312\end{array}$ & $\stackrel{-}{0.303}$ & $\begin{array}{c}- \\
0.707\end{array}$ \\
\hline CC vsTT & $\begin{array}{c}7[9,10,17,18,21] \\
\text { a6 }[9,10,17,18,21]\end{array}$ & $\begin{array}{l}0.62(0.49 \sim 0.77) \\
0.67(0.61 \sim 0.73)\end{array}$ & $\begin{array}{l}4.28 \\
8.29\end{array}$ & $\begin{array}{l}<0.001 \\
<0.001\end{array}$ & $\begin{array}{c}72.2 \% \\
0.0 \%\end{array}$ & $\begin{array}{l}0.001 \\
0.540\end{array}$ & $\stackrel{-}{0.520}$ & 1.000 \\
\hline \multicolumn{9}{|c|}{ rs2735839 } \\
\hline A allele vs $G$ allele & $\begin{array}{c}14[8,12,14,17,19-22,25,26] \\
\text { b11 }[8,14,17,19-22,25,26]\end{array}$ & $\begin{array}{l}0.78(0.71 \sim 0.86) \\
0.86(0.82 \sim 0.90)\end{array}$ & $\begin{array}{l}4.96 \\
6.35\end{array}$ & $\begin{array}{l}<0.001 \\
<0.001\end{array}$ & $\begin{array}{l}87.5 \% \\
36.4 \%\end{array}$ & $\begin{array}{c}<0.001 \\
0.108\end{array}$ & $\frac{-}{0.152}$ & 0.533 \\
\hline$A G$ vs $G G$ & $\begin{array}{c}10[12,14,17,19,21,26] \\
\text { b7 }[14,17,19,21,26]\end{array}$ & $\begin{array}{l}0.80(0.71 \sim 0.91) \\
0.85(080 \sim 0.90)\end{array}$ & $\begin{array}{l}3.52 \\
5.50\end{array}$ & $\begin{array}{c}0.001 \\
<0.001\end{array}$ & $\begin{array}{l}87.3 \% \\
36.7 \%\end{array}$ & $\begin{array}{c}<0.001 \\
0.148\end{array}$ & $\begin{array}{c}- \\
0.424\end{array}$ & $\begin{array}{c}- \\
1.000\end{array}$ \\
\hline$A A$ vs $G G$ & $\begin{array}{c}10[12,14,17,19,21,26] \\
\text { b7 }[14,17,19,21,26]\end{array}$ & $\begin{array}{l}0.77(0.54 \sim 1.10) \\
0.81(0.67 \sim 0.97)\end{array}$ & $\begin{array}{l}1.46 \\
2.32\end{array}$ & $\begin{array}{l}0.144 \\
0.020\end{array}$ & $\begin{array}{l}88.1 \% \\
39.0 \%\end{array}$ & $\begin{array}{c}<0.001 \\
0.131\end{array}$ & $0 . \overline{158}$ & $0 . \overline{230}$ \\
\hline \multicolumn{9}{|c|}{ rs266882 } \\
\hline A allele vs G allele & $\begin{array}{c}4[15,18,27,28] \\
\text { c2 }[18,28]\end{array}$ & $\begin{array}{l}1.26(0.97 \sim 1.64) \\
1.00(0.91 \sim 1.10)\end{array}$ & $\begin{array}{l}1.71 \\
0.01\end{array}$ & $\begin{array}{l}0.087 \\
0.995\end{array}$ & $\begin{array}{c}83.7 \% \\
0.0 \%\end{array}$ & $\begin{array}{c}<0.001 \\
0.978\end{array}$ & - & $\begin{array}{l}- \\
-\end{array}$ \\
\hline$A G$ vs $G G$ & $\begin{array}{c}4[15,18,27,28] \\
\text { d3 }[15,18,28]\end{array}$ & $\begin{array}{l}1.40(0.92 \sim 2.13) \\
1.20(0.82 \sim 1.76)\end{array}$ & $\begin{array}{l}1.56 \\
0.95\end{array}$ & $\begin{array}{l}0.119 \\
0.340\end{array}$ & $\begin{array}{l}68.2 \% \\
57.1 \%\end{array}$ & $\begin{array}{l}0.024 \\
0.097\end{array}$ & $\begin{array}{c}- \\
0.622\end{array}$ & $\begin{array}{c}- \\
0.296\end{array}$ \\
\hline$A A$ vs $G G$ & $\begin{array}{c}4[15,18,27,28] \\
\text { d3 }[15,18,28]\end{array}$ & $\begin{array}{l}1.45(0.92 \sim 2.29) \\
1.18(0.80 \sim 1.72)\end{array}$ & $\begin{array}{l}1.59 \\
0.84\end{array}$ & $\begin{array}{l}0.112 \\
0.402\end{array}$ & $\begin{array}{l}74.4 \% \\
62.9 \%\end{array}$ & $\begin{array}{l}0.008 \\
0.068\end{array}$ & $0 . \overline{277}$ & $0 . \overline{296}$ \\
\hline \multicolumn{9}{|c|}{ rs 174776} \\
\hline T allele vs $C$ allele & $3[16,18,21]$ & $0.86(0.80 \sim 0.93)$ & 3.74 & $<0.001$ & $0.0 \%$ & 0.619 & 0.326 & 1.000 \\
\hline CT vs CC & $2[18,21]$ & $0.87(0.79 \sim 0.97)$ & 2.58 & 0.010 & $0.0 \%$ & 0.844 & - & - \\
\hline TT vs CC & $2[18,21]$ & $0.77(0.55 \sim 1.06)$ & 1.62 & 0.106 & $0.0 \%$ & 0.436 & - & - \\
\hline \multicolumn{9}{|c|}{ rs17632542 } \\
\hline T allele vs C allele & $\begin{array}{c}4[17,22] \\
\text { a3 }[17,22]\end{array}$ & $\begin{array}{l}0.61(0.43 \sim 0.86) \\
0.72(0.64 \sim 0.82)\end{array}$ & $\begin{array}{l}2.79 \\
4.99\end{array}$ & $\begin{array}{l}0.005 \\
<0.001\end{array}$ & $\begin{array}{l}95.0 \% \\
50.2 \%\end{array}$ & $\begin{array}{c}<0.001 \\
0.134\end{array}$ & $\stackrel{-}{0.659}$ & $\frac{-}{1.000}$ \\
\hline TC vs CC & $\begin{array}{c}3[17] \\
\text { a2 [17] }\end{array}$ & $\begin{array}{l}0.57(0.37 \sim 0.87) \\
0.72(0.59 \sim 0.87)\end{array}$ & $\begin{array}{l}2.61 \\
3.43\end{array}$ & $\begin{array}{l}0.009 \\
0.001\end{array}$ & $\begin{array}{l}95.4 \% \\
72.2 \%\end{array}$ & $\begin{array}{c}<0.001 \\
0.058\end{array}$ & - & $\begin{array}{l}- \\
-\end{array}$ \\
\hline TT vs CC & $\begin{array}{c}3[17] \\
\text { a2 [17] }\end{array}$ & $\begin{array}{l}0.32(0.14 \sim 0.75) \\
0.50(0.30 \sim 0.83)\end{array}$ & $\begin{array}{l}2.62 \\
2.68\end{array}$ & $\begin{array}{l}0.009 \\
0.007\end{array}$ & $\begin{array}{c}73.1 \% \\
0.0 \%\end{array}$ & $\begin{array}{l}0.024 \\
0.819\end{array}$ & $\begin{array}{l}- \\
-\end{array}$ & $\begin{array}{l}- \\
-\end{array}$ \\
\hline
\end{tabular}


Table 3. Meta-analysis of associations between SNPs and PCa risk - cont.

\begin{tabular}{|c|c|c|c|c|c|c|c|c|}
\hline \multirow{2}{*}{ SNP } & \multirow{2}{*}{ Number of studies } & \multicolumn{3}{|c|}{ Test for overall effect } & \multicolumn{2}{|c|}{ Test for heterogeneity } & \multicolumn{2}{|c|}{ Test for publish bias } \\
\hline & & OR $(95 \% \mathrm{Cl})$ & Z-score & p-value & $1^{2}$ & $\mathrm{p}$-value & Pegger's & $P_{\text {begg's }}$ \\
\hline \multicolumn{9}{|c|}{ rs266849 } \\
\hline G allele vs A allele & $\begin{array}{c}8[17,19,26] \\
\text { e5 }[17,19,26]\end{array}$ & $\begin{array}{l}0.81(0.71 \sim 0.92) \\
0.94(0.89 \sim 0.98)\end{array}$ & $\begin{array}{l}3.16 \\
2.68\end{array}$ & $\begin{array}{l}0.002 \\
0.007\end{array}$ & $\begin{array}{l}92.2 \% \\
17.0 \%\end{array}$ & $\begin{array}{c}<0.001 \\
0.306\end{array}$ & $\begin{array}{c}- \\
0.289\end{array}$ & $\begin{array}{c}- \\
0.462\end{array}$ \\
\hline GA vs AA & $\begin{array}{c}7[17,19,26] \\
\text { e4 }[17,19,26]\end{array}$ & $\begin{array}{l}0.80(0.70 \sim 0.91) \\
0.91(0.85 \sim 0.98)\end{array}$ & $\begin{array}{l}3.40 \\
2.55\end{array}$ & $\begin{array}{l}0.001 \\
0.011\end{array}$ & $\begin{array}{l}90.1 \% \\
43.8 \%\end{array}$ & $\begin{array}{c}<0.001 \\
0.149\end{array}$ & $\begin{array}{c}- \\
0.927\end{array}$ & $\begin{array}{c}- \\
0.734\end{array}$ \\
\hline GG vs $A A$ & $\begin{array}{c}7[17,19,26] \\
\text { e4 }[17,19,26]\end{array}$ & $\begin{array}{l}0.73(0.55 \sim 0.97) \\
0.98(0.86 \sim 1.10)\end{array}$ & $\begin{array}{l}2.15 \\
0.39\end{array}$ & $\begin{array}{l}0.032 \\
0.699\end{array}$ & $\begin{array}{c}87.2 \% \\
4.4 \%\end{array}$ & $\begin{array}{c}<0.001 \\
0.371\end{array}$ & $\begin{array}{c}- \\
0.812\end{array}$ & 1.000 \\
\hline \multicolumn{9}{|c|}{$r s 266878$} \\
\hline G allele vs $C$ allele & $2[18,21]$ & $0.86(0.78 \sim 0.94)$ & 3.23 & 0.001 & $0.0 \%$ & 0.410 & - & - \\
\hline GC vs CC & $2[18,21]$ & $0.87(0.78 \sim 0.97)$ & 2.60 & 0.009 & $0.0 \%$ & 1.000 & - & - \\
\hline GG vs CC & $2[18,21]$ & $0.72(0.52 \sim 0.98)$ & 2.10 & 0.036 & $0.0 \%$ & 0.354 & - & - \\
\hline \multicolumn{9}{|c|}{ rs266876 } \\
\hline Callele vs T allele & $2[18,21]$ & $0.83(0.63 \sim 1.08)$ & 1.42 & 0.157 & $90.9 \%$ & 0.001 & - & - \\
\hline CT vsTT & $2[18,21]$ & 0.99 (0.90 1.08) & 0.22 & 0.825 & $0.0 \%$ & 0.703 & - & - \\
\hline CC vs TT & $2[18,21]$ & $0.77(0.65 \sim 0.91)$ & 3.02 & 0.003 & $0.0 \%$ & 0.784 & - & - \\
\hline \multicolumn{9}{|c|}{ rs1058274 } \\
\hline G allele vs $A$ allele & $2[18,21]$ & $0.98(0.92 \sim 1.05)$ & 0.56 & 0.578 & $0.0 \%$ & 0.669 & - & - \\
\hline GA vs AA & $2[18,21]$ & $1.01(0.92 \sim 1.11)$ & 0.15 & 0.878 & $0.0 \%$ & 0.926 & - & - \\
\hline GG vs $A A$ & $2[18,21]$ & $0.94(0.82 \sim 1.09)$ & 0.81 & 0.419 & $0.0 \%$ & 0.658 & - & - \\
\hline \multicolumn{9}{|c|}{ rs2569735 } \\
\hline A allele vs $G$ allele & $2[18,21]$ & $0.90(0.82 \sim 0.99)$ & 2.14 & 0.032 & $8.4 \%$ & 0.296 & - & - \\
\hline$A G$ vs GG & $2[18,21]$ & $0.92(0.83 \sim 1.02)$ & 1.61 & 0.108 & $0.0 \%$ & 0.464 & - & - \\
\hline$A A$ vs $G G$ & $2[18,21]$ & $0.72(0.52 \sim 0.99)$ & 2.03 & 0.042 & $0.0 \%$ & 0.583 & - & - \\
\hline
\end{tabular}

a The heterogeneity test showed that the data of Kote-Jarai et al. ${ }^{17}$ (stage 1) was heterogeneous. After excluding it, the heterogeneity was eliminated; ${ }^{b}$ The heterogeneity test showed that the data of Kote-Jarai et al. ${ }^{17}$ (stage 1), Eeles et al. ${ }^{26}$ (stage 1) and Hu et al. ${ }^{12}$ was heterogeneous. After excluding it, the heterogeneity was eliminated; ${ }^{~}$ The heterogeneity test showed that the data of Soni et al. ${ }^{15}$ and Lai et al. ${ }^{27}$ was heterogeneous. After excluding it, the heterogeneity was eliminated; ${ }^{d}$ The heterogeneity test showed that the data of Lai et al. ${ }^{27}$ was heterogeneous. After excluding it, the heterogeneity was eliminated; ${ }^{\text {e }}$ The heterogeneity test showed that the data of Kote-Jarai et al. ${ }^{17}$ (stage 1), Kote-Jarai et al. ${ }^{17}$ (stage 3 ) and Eeles et al. ${ }^{26}$ (stage 1 ) was heterogeneous. After excluding it, the heterogeneity was eliminated.

SNP - single nucleotide polymorphism; PCa - prostate cancer; $95 \% \mathrm{Cl}$ - 95\% confidence interval. Kote-Jarai et al. ${ }^{17}$ was identified as 3 studies (stage 1 , stage 2 and stage 3); Eeles et al. ${ }^{26}$ was also identified as 3 studies (stage 1, stage 2 UK and stage 2 Australia).

associated with the risk of PCa (rs1058205 C vs T allele: $\mathrm{OR}=0.79,95 \% \mathrm{CI}=0.73 \sim 0.87$, p-value $<0.001 ;$ rs 2735839 A vs $\mathrm{G}$ allele: $\mathrm{OR}=0.78,95 \% \mathrm{CI}=0.71 \sim 0.86$, p-value $<0.001$; rs174776 T vs $\mathrm{C}$ allele: $\mathrm{OR}=0.86,95 \% \mathrm{CI}=0.80 \sim 0.93$, p-value $<0.001$; rs17632542 $\mathrm{T}$ vs $\mathrm{C}$ allele: $\mathrm{OR}=0.61$, $95 \% \mathrm{CI}=0.43 \sim 0.86$, p-value $=0.005 ;$ rs $266849 \mathrm{G}$ vs A allele: $\mathrm{OR}=0.81,95 \% \mathrm{CI}=0.71 \sim 0.92$, $\mathrm{p}$-value $=0.002$; rs266878 G vs C allele: $\mathrm{OR}=0.86,95 \% \mathrm{CI}=0.78 \sim 0.94$, $\mathrm{p}$-value $=0.001 ; \operatorname{rs} 2569735 \mathrm{~A}$ vs $\mathrm{G}: \mathrm{OR}=0.90$, $95 \% \mathrm{CI}=0.82 \sim 0.99$, $\mathrm{p}$-value $=0.032$ ). For the genotypes, the pooled results showed that the genotype TC (TC vs TT: $\mathrm{OR}=0.79,95 \% \mathrm{CI}=0.72 \sim 0.86, \mathrm{p}$-value $<0.001)$ and $\mathrm{CC}$ (CC vs TT: $\mathrm{OR}=0.62,95 \% \mathrm{CI}=0.49 \sim 0.77$, p-value $<0.001$ ) of rs1058205, the genotype AG (AG vs GG: OR =0.80, $95 \% \mathrm{CI}=0.71 \sim 0.91$, p-value $=0.001)$ of rs2735839, the genotype CT (CT vs CC: $\mathrm{OR}=0.87,95 \% \mathrm{CI}=0.79 \sim 0.97$, $\mathrm{p}$-value $=0.010)$ of rs174776, the genotype TC (TC vs CC: $\mathrm{OR}=0.57,95 \% \mathrm{CI}=0.37 \sim 0.87$, p-value $=0.009)$ and $\mathrm{TT}$ (TT vs CC: $\mathrm{OR}=0.32,95 \% \mathrm{CI}=0.14 \sim 0.75$, p-value $=0.009$ ) of rs17632542, the genotype GA (GA vs AA: OR $=0.80$,
95\% CI $=0.70 \sim 0.91, \mathrm{p}$-value $=0.001)$ and $\mathrm{GG}$ (GG vs AA: $\mathrm{OR}=0.73,95 \% \mathrm{CI}=0.55 \sim 0.97, \mathrm{p}$-value $=0.032)$ of rs266849, the genotype GC (GC vs CC: $\mathrm{OR}=0.87$, 95\% CI $=0.78 \sim 0.97$, p-value $=0.009)$ and $\mathrm{GG}$ (GG vs CC: $\mathrm{OR}=0.72,95 \% \mathrm{CI}=0.52 \sim 0.98$, $\mathrm{p}$-value $=0.036)$ of $\mathrm{rs} 266878$, the genotype $\mathrm{CC}$ (CC vs TT: $\mathrm{OR}=0.77,95 \% \mathrm{CI}=0.65 \sim 0.91$, $\mathrm{p}$-value $=0.003)$ of rs266876, and the genotype AA (AA vs $\mathrm{GG}$ : $\mathrm{OR}=0.72,95 \% \mathrm{CI}=0.52 \sim 0.99, \mathrm{p}$-value $=0.042$ ) of rs2569735 were statistically associated with PCa risk, while there was no significance for the genotype AA of rs2735839, the genotype AG and AA of rs266882, the genotype TT of rs174776, the genotype CT of rs266876, the genotype GA and GG of rs1058274, or the genotype AG of rs2569735.

\section{Meta-analysis of associations between SNPs of KLK3 and the Gleason score of PCa}

Only rs2735839 was involved in the meta-analysis of associations between SNPs of $K L K 3$ and the Gleason score 
Table 4. Meta-analysis for associations between SNPs and the GS of PCa

\begin{tabular}{|c|c|c|c|c|c|c|c|c|}
\hline \multirow{2}{*}{ SNP } & \multirow{2}{*}{$\begin{array}{l}\text { Number } \\
\text { of studies }\end{array}$} & \multicolumn{3}{|c|}{ Test for overall effect } & \multicolumn{2}{|c|}{ Test for heterogeneity } & \multicolumn{2}{|c|}{ Test for publish bias } \\
\hline & & OR $(95 \% \mathrm{Cl})$ & Z-score & p-value & $1^{2}$ & $p$-value & $P_{\text {egger's }}$ & $P_{\text {begg's }}$ \\
\hline \multicolumn{9}{|c|}{ rs2735839GS $<7$ vs GS $\geq 8$} \\
\hline A allele vs G allele & $3[12,24]$ & $0.598(0.465 \sim 0.770)$ & 3.99 & $<0.001$ & $0.0 \%$ & 0.806 & - & - \\
\hline AG/GG vs AA & $2[11,12]$ & $2.731(0.622 \sim 12.00)$ & 1.33 & 0.183 & $75.5 \%$ & 0.043 & - & - \\
\hline \multicolumn{9}{|c|}{ rs2735839 GS < 8 vs control } \\
\hline G allele vs A allele & $2[19,21]$ & $0.841(0.796 \sim 0.889)$ & 6.14 & $<0.001$ & $0.0 \%$ & 0.883 & - & - \\
\hline \multicolumn{9}{|c|}{ rs2735839 GS $\geq 8$ vs control } \\
\hline G allele vs A allele & $2[19,21]$ & $1.09(0.991 \sim 1.201)$ & 1.77 & 0.077 & $0.0 \%$ & 0.517 & - & - \\
\hline \multicolumn{9}{|c|}{$r s 2735839$ GS $\geq 4+3$ vs GS $\leq 3+4$} \\
\hline G allele vs A allele & $2[11,24]$ & $1.413(1.257 \sim 1.588)$ & 5.80 & $<0.001$ & $0.0 \%$ & 0.360 & - & - \\
\hline
\end{tabular}

SNP - single nucleotide polymorphism; GS - Gleason score; PCa - prostate cancer; $95 \% \mathrm{Cl}$ - 95\% confidence interval.

(GS) of PCa. ${ }^{11,12,19,21,24}$ As shown in Table 4, when compared with the group of $\mathrm{GS} \geq 8$ carrier, the A allele was a protective factor for the group of GS $<7$ (A vs $G$ allele: $\mathrm{OR}=0.598,95 \% \mathrm{CI}=0.465 \sim 0.770$, $\mathrm{p}$-value $<0.001$ ); when compared with the group of $\mathrm{GS} \leq 3+4$ carrier, the $\mathrm{G}$ allele was a risk factor for the group of $G S \geq 4+3$ ( $G$ vs A allele: $\mathrm{OR}=1.413,95 \% \mathrm{CI}=1.257 \sim 1.588$, p-value $<0.001)$. When compared with the controls, the $\mathrm{G}$ allele was a protective factor for the group of GS $<8$ (G vs A allele: $\mathrm{OR}=0.841$, $95 \% \mathrm{CI}=0.796 \sim 0.889$, p-value $<0.001)$, while not significantly associated with the group of GS $\geq 8$ (G vs A allele: $\mathrm{OR}=1.09,95 \% \mathrm{CI}=0.991 \sim 1.201$, p-value $<0.077)$.

\section{Meta-analysis for associations between SNPs of KLK3 and fatal PCa risk}

SNP rs2735839 was also involved in the meta-analysis of associations between SNPs and the risk of fatal PCa. ${ }^{13,23}$ The pooled result showed that there was no significance between rs2735839 and fatal PCa (G vs A allele: $\mathrm{OR}=1.230$, 95\% CI $=0.725 \sim 2.088$, p-value $=0.442$ ).

\section{Heterogeneity test and sensitivity analysis}

A heterogeneity test was performed and the results showed that heterogeneity existed in the meta-analysis of associations between the risk of PCa and rs1058205, rs2735839, rs266882, rs17632542, and rs266849. Therefore, a sensitivity analysis was conducted employing the sequential omission of individual studies to find the source of the heterogeneity. As shown in Table 3, after excluding some studies, the heterogeneity was eliminated. Most pooled results were not materially altered, indicating the robustness of the results of this meta-analysis, except the meta-analysis of the genotype (AA vs GG) of rs2735839 and the genotype (GG vs AA) of rs266849. After eliminating the heterogeneity, the genotype AA of rs2735839 was significantly associated with PCa risk (AA vs GG:
$\mathrm{OR}=0.81,95 \% \mathrm{CI}=0.67 \sim 0.97, \mathrm{p}$-value $=0.020)$, while there was no significant association between the genotype GG of rs266849 and PCa risk (GG vs AA: OR = 0.98, $95 \% \mathrm{CI}=0.86 \sim 1.10$, $\mathrm{p}$-value $=0.699$ ).

\section{Publication bias assessment}

Begg's funnel plot and Egger's test were performed to assess publication bias in the literature if the number of included studies was more than 3. The results of this meta-analysis showed that no evidence of publication bias was found for any of the analyses.

\section{Discussion}

The etiology and pathogenesis of $\mathrm{PCa}$ is still elusive. However, recently, increasing evidence suggests that genetic factors are associated with PCa susceptibility. For many years, PSA, which plays an important role in sperm motility, has been used as a biomarker for PCa screening. The PSA is also involved in the proteolytic breakdown of the extracellular matrix in PCa tumorigenesis, which contributes to tumor invasion and metastasis ${ }^{29}$; high serum PSA correlates with mutations in p53 and overexpression of the B-cell lymphoma 2 protein, which inhibits apoptosis in tumor cells. ${ }^{30}$ These findings strongly suggest that PSA plays a role in the etiology of $\mathrm{PCa}$. The PSA protein is encoded by $K L K 3$, and increasing numbers of studies have recently reported that the polymorphisms of $K L K 3$ associated with PSA levels may be associated with PCa. However, these results were conflicting and there was still no comprehensive analysis to clear up the confusion. Therefore, in this study, we performed a literature review and conducted a meta-analysis to explore the association between the SNPs of $K L K 3$ that were analyzed in more than 2 studies and the risk of PCa.

In total, 59 SNPs were mentioned in the literature, and among them, 21 SNPs were involved in more than 
2 studies. Finally, 10 SNPs - rs1058205, rs2735839, rs266882, rs174776, rs17632542, rs266849, rs266878, rs266876, rs1058274, and rs2569735 - were eligible to be included in this meta-analysis. The pooled results indicated that the minor alleles of rs1058205 (C allele), rs2735839 (A allele), rs174776 ( $\mathrm{T}$ allele), rs17632542 (T allele), rs266849 (G allele), rs266878 (G allele), and rs2569735 (A allele) were significantly associated with PCa risk. For the genotype analysis, when compared to genotypes of the common homozygotes (rs1058205: TT, rs2735839: GG, rs174776: CC, rs17632542: CC, rs266849:AA, rs266878: CC, rs266876: TT, and rs2569735: GG), the heterozygote genotype carriers of rs1058205 (CT), rs2735839 (AG), rs174776 (CT), rs17632542 (TC), rs266849 (GA), and rs266878 (GC) had a lower risk of $\mathrm{PCa}$, as did the homozygotes genotype carrier of rs1058205 (CC), rs2735839 (AA), rs17632542 (TT), rs266878 (GG), rs266876 (CC), and rs2569735 (AA).

The Gleason grading system remains the most powerful prognostic predictor for PCa because it delineates the architectural patterns of tumors. ${ }^{31}$ It is the core value in riskscoring systems, including the D'Amico classification system, ${ }^{32}$ which incorporates the GS, clinical stage and PSA level to stratify the risk of recurrence of localized PCa before treatment and is used to guide treatment selection. Thus, we subsequently performed the GS striated analyses; only rs2735839 was involved in this part. The GS results range from 1 to 10 , and can be divided into 3 grades: GS 1-6 is the low grade in the Gleason grading system; GS 8-10 is the high grade; and GS 7 is the intermediate grade. Our pooled results showed that when compared with $\mathrm{GS} \geq 8 \mathrm{PCa}$ (high grade), the A allele was a protective factor for GS $<7 \mathrm{PCa}$. Patients with GS 7 PCa are a heterogeneous group, consisting of 2 subtypes: GS $3+4$ and GS $4+3$. $^{33}$ The GS 4+3 subtype has had less favorable clinical outcomes than the GS 3+4 subtype. ${ }^{33-35}$ Therefore, the GS $3+4$ subtype can be treated as low grade, while GS $4+3$ subtype is high grade. Currently there are no reliable biomarkers to further stratify this group. Some studies have therefore stratified GS 7 PCa to explore effective biomarkers. We pooled their relevant data, and the results indicated that when compared to the GS $\leq 3+4$ PCa carrier, the G allele was a risk factor for the $\mathrm{GS} \geq 4+3$ carrier.

Finally, we also performed a meta-analysis of associations of the SNPs of $K L K 3$ and fatal PCa, and again only rs2735839 was involved in this analysis. Our pooled results suggested that there was no significant association between them.

To our knowledge, this is the first study to review all of the SNPs of $K L K 3$ mentioned in the relevant literature and to perform meta-analyses to illuminate the association between the risk of PCa and SNPs of $K L K 3$ that have been involved in more than 2 studies. Although our study showed some positive results, this meta-analysis had 2 limitations that should be taken into consideration when assessing the results. First, the overall outcomes were based on unadjusted effect estimates. Among the included studies, only a few were matched for age or other factors.
Therefore, some other confounding factors could slightly modify the estimates, and a more precise evaluation would have to be adjusted for the potentially suspicious factors. Second, in some pooled analyses such as the GS analysis, the number of included studies was too small, so further relevant studies should be carried out in the future so that a stronger conclusion can be drawn.

\section{Conclusions}

A strong association was observed between rs1058205, rs2735839, rs266882, rs174776, rs17632542, rs266849, rs266878, rs266876, rs1058274 and rs2569735, and PCa. Therefore, these SNPs may be valuable as biomarkers for PCa risk. Besides, G allele of rs2735839 was noted as a risk factor for the GS $<7$ PCa carrier when compared with GS $\geq 8 \mathrm{PCa}$, as well as for the GS $\geq 4+3$ carrier when compared to the GS $\leq 3+4 \mathrm{PCa}$ carrier. Considering that the quality and quantity of the reviewed articles were limited, larger well-designed studies should be conducted in the future to further confirm the association between KLK3 genetic polymorphisms and $\mathrm{PCa}$.

\section{References}

1. Askari F, Parizi MK, Jessri M, Rashidkhani B. Fruit and vegetable intake in relation to prostate cancer in Iranian men: A case-control study. Asian Pac J Cancer Prev. 2014;15(13):5223-5227.

2. Altekruse SF, Huang L, Cucinelli JE, McNeel TS, Wells KM, Oliver MN. Spatial patterns of localized-stage prostate cancer incidence among white and black men in the southeastern United States. Cancer Epidemiol Biomarkers Prev. 2010;19(6):1460-1467.

3. Pilia G, Chen WM, Scuteri A, et al. Heritability of cardiovascular and personality traits in 6,148 Sardinians. PLoS Genet. 2006;2(8):e132.

4. Bansal A, Murray DK, Wu JT, Stephenson RA, Middleton RG, Meikle AW. Heritability of prostate-specific antigen and relationship with zonal prostate volumes in aging twins. J Clin Endocrinol Metab. 2000;85(3): 1272-1276.

5. Ahn J, Berndt SI, Wacholder S, et al. Variation in KLK genes, prostatespecific antigen and risk of prostate cancer. Nat Genet. 2008;40(9): 1032-1034.

6. Wiklund F, Zheng SL, Sun J, et al. Association of reported prostate cancer risk alleles with PSA levels among men without a diagnosis of prostate cancer. Prostate. 2009;69(4):419-427.

7. Eeles RA, Kote-Jarai Z, Giles GG, et al. Multiple newly identified loci associated with prostate cancer susceptibility. Nat Genet. 2008;40(3): 316-321.

8. Choe EK, Lee Y, Cho JY, et al. Search for genetic factor association with cancer-free prostate-specific antigen level elevation on the basis of a genome-wide association study in the Korean population. Eur J Cancer Prev. 2017;27(5):453-460. doi:10.1097/CEJ.0000000000000359

9. Chen C, Xin Z. Single-nucleotide polymorphism rs 1058205 of KLK3 is associated with the risk of prostate cancer: A case-control study of Han Chinese men in Northeast China. Medicine (Baltimore). 2017; 96(10):e6280.

10. Stegeman S, Amankwah E, Klein K, et al. A large-scale analysis of genetic variants within putative miRNA binding sites in prostate cancer. Cancer Discov. 2015;5(4):368-379.

11. He Y, Gu J, Strom S, Logothetis CJ, Kim J, Wu X. The prostate cancer susceptibility variant rs 2735839 near KLK3 gene is associated with aggressive prostate cancer and can stratify Gleason score 7 patients. Clin Cancer Res. 2014;20(19):5133-5139.

12. Hu J, Qiu Z, Zhang L, Cui F. Kallikrein 3 and vitamin D receptor polymorphisms: Potentials environmental risk factors for prostate cancer. Diagn Pathol. 2014; 9:84. 
13. Shui IM, Lindström S, Kibel AS, et al. Prostate cancer (PCa) risk variants and risk of fatal PCa in the National Cancer Institute Breast and Prostate Cancer Cohort Consortium. Eur Urol. 2014(6);65:1069-1075.

14. Wang NN, Xu Y, Yang K, et al. Susceptibility loci associations with prostate cancer risk in Northern Chinese men. Asian Pac J Cancer Prev. 2013;14(5):3075-3078.

15. Soni A, Bansal A, Mishra AK, et al. Association of androgen receptor, prostate-specific antigen, and CYP19 gene polymorphisms with prostate carcinoma and benign prostatic hyperplasia in a North Indian population. Genet Test Mol Biomarkers. 2012;16(8):835-840.

16. Kwon EM, Holt SK, Fu R, et al. Androgen metabolism and JAK/STAT pathway genes and prostate cancer risk. Cancer Epidemiol. 2012;36(4): 347-353.

17. Kote-Jarai Z, Amin Al Olama A, et al. Identification of a novel prostate cancer susceptibility variant in the KLK3 gene transcript. Hum Genet. 2011;129(6):687-694.

18. Penney KL, Schumacher FR, Kraft P, et al. Association of KLK3 (PSA) genetic variants with prostate cancer risk and PSA levels. Carcinogenesis. 2011;32(6):853-859.

19. Lindstrom S, Schumacher F, Siddiq A, et al. Characterizing associations and SNP-environment interactions for GWAS-identified prostate cancer risk markers-results from BPC3. PLoS One. 2011;6(2):e17142.

20. Ciampa J, Yeager M, Amundadottir L, et al. Large-scale exploration of gene-gene interactions in prostate cancer using a multistage genome-wide association study. Cancer Res. 2011;71(9):3287-3295.

21. Parikh $\mathrm{H}$, Wang $\mathrm{Z}$, Pettigrew KA, et al. Fine mapping the KLK3 locus on chromosome 19q13.33 associated with prostate cancer susceptibility and PSA levels. Hum Genet. 2011;129(6):675-685.

22. Gudmundsson J, Besenbacher S, Sulem P, et al. Genetic correction of PSA values using sequence variants associated with PSA levels. Sci Transl Med. 2010;2(62):62ra92.

23. Gallagher DJ, Vijai J, Cronin AM, et al. Susceptibility loci associated with prostate cancer progression and mortality. Clin Cancer Res. 2010;16(10):2819-2832.

24. Kader AK, Sun J, Isaacs SD, et al. Individual and cumulative effect of prostate cancer risk-associated variants on clinicopathologic variables in 5,895 prostate cancer patients. Prostate. 2009;69(11):1195-1205.
25. Xu J, Isaacs SD, Sun J, et al. Association of prostate cancer risk variants with clinicopathologic characteristics of the disease. Clin Cancer Res. 2008;14(18):5819-5824.

26. Eeles RA, Kote-Jarai Z, Giles GG, et al. Multiple newly identified loci associated with prostate cancer susceptibility. Nat Genet. 2008;40(3): 316-321.

27. Lai J, Kedda MA, Hinze K, et al. PSA/KLK3 AREl promoter polymorphism alters androgen receptor binding and is associated with prostate cancer susceptibility. Carcinogenesis. 2007;28(5):1032-1039.

28. Cicek MS, Liu X, Casey G, Witte JS. Role of androgen metabolism genes CYP1B1, PSA/KLK3, and CYP11alpha in prostate cancer risk and aggressiveness. Cancer Epidemiol Biomarkers Prev. 2005;14(9): 2173-2177.

29. Webber MM, Waghray A, BelloD. Prostate-specific antigen, a serine protease, facilitates human prostate cancer cell invasion. Clin Cancer Res. 1995;1(10):1089-1094.

30. Lin JT, Wang JS, Jiann BP, et al. Correlation of p53 protein accumulation and $\mathrm{BCl}-2$ overexpression with histopathological features in prostatic cancer. J Formos Med Assoc. 2005;104(11):864-867.

31. Epstein JI, Allsbrook WC Jr. Amin MB, Egevad LL. The 2005 International Society of Urological Pathology (ISUP) Consensus Conference on Gleason Grading of Prostatic Carcinoma. Am J Surg Pathol. 2005;29(9):1228-1242.

32. D'Amico AV, Whittington R, Malkowicz SB, et al. Biochemical outcome after radical prostatectomy, external beam radiation therapy, or interstitial radiation therapy for clinically localized prostate cancer. JAMA. 1998;280(11):969-974.

33. Orozco R, O'Dowd G, Kunnel B, Miller MC, Veltri RW. Observations on pathology trends in 62,537 prostate biopsies obtained from urology private practices in the United States. Urology. 1998;51(2):186-195.

34. Sakr WA, Tefilli MV, Grignon DJ, et al. Gleason score 7 prostate cancer: A heterogeneous entity? Correlation with pathologic parameters and disease-free survival. Urology. 2000;56(5):730-734.

35. Chan TY, Partin AW, Walsh PC, Epstein JI. Prognostic significance of Gleason score 3+4 versus Gleason score $4+3$ tumor at radical prostatectomy. Urology. 2000;56(5):823-827. 